\title{
What you show is what you get: sampling biases in determining biological sensory function
}

\section{Alexander G Dimitrov}

Address: Center for Computational Biology, Montana State University, Bozeman, MT 59715, USA

Email: Alexander G Dimitrov - alex@cns.montana.edu

from Seventeenth Annual Computational Neuroscience Meeting: CNS*2008

Portland, OR, USA. 19-24 July 2008

Published: II July 2008

BMC Neuroscience 2008, 9(SuppI I):PI2I doi:I0.1 I86/I47|-2202-9-SI-PI2I

This abstract is available from: http://www.biomedcentral.com/I47I-2202/9/SI/PI2 I

(c) 2008 Dimitrov; licensee BioMed Central Ltd.

\section{Introduction}

Classical studies of biological sensory systems use the following main technique: sensory stimuli are drawn from a pre-determined distribution $\mathrm{P}($ stim) and presented to the animal; the ensemble associated with sensory response is collected and used to characterize the conditional distribution P(stim|resp) (or parameters thereof) as a model of sensory system function. However, most of the standard statistical tool used in neuroscience to estimate $\mathrm{P}($ stim|resp) are valid under a very fundamental condi-

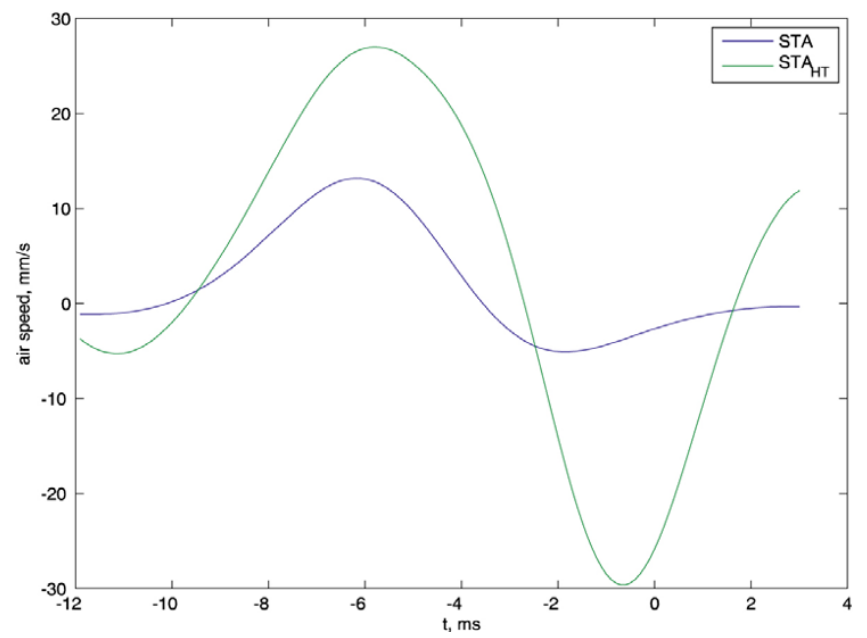

\section{Figure I}

The spike-triggered average (STA) of air current stimulis sampled from a band-limited GWN distribution $(5-500 \mathrm{~Hz})$ is shown in blue. The corresponding Horvitz-Thompson estimate of the STA is plotted in green. tion - that the samples used to estimate $\mathrm{P}($ stim|resp) are drawn from the same distribution. This is obviously not the case in most studies of sensory system, where the samples are drawn explicitly from a different distribution, $\mathrm{P}(\mathrm{stim})$ (the sampling distribution), selected by the scientist. We demonstrate here that in this case the observed conditional distribution is $\mathrm{P}^{*}(\mathrm{stim} \mid \mathrm{resp})=\mathrm{P}($ stim $\mid$ resp $)$ ${ }^{*} \mathrm{P}(\mathrm{stim})$ and expectations estimated with this dataset are parameters of $\mathrm{P}^{*}$, not $\mathrm{P}$. To characterize the actual functional properties of the system, one needs to use estimators developed within unequal probability sampling theory [1]. We apply one of these estimators, the HorvitzThompson estimator of the mean $\mathrm{m}_{\mathrm{HT}}=\Sigma_{\mathrm{i}} \mathrm{x}_{\mathrm{i}} / \mathrm{P}\left(\mathrm{x}_{\mathrm{i}}\right)$, to observations $\left\{\mathrm{x}_{\mathrm{i}}\right\}$ from the cricket cercal sensory system and illustrate the ensuing changes in apparent functionality (Figure 1)

\section{References}

I. Thomson SK: Sampling 2nd edition. New York: Wiley Interscience; 2002. 